\title{
Adsorption and reaction processes of physisorbed molecular oxygen on $\mathrm{Si}(111)-(7 \times 7)$
}

\author{
Kazuyuki Sakamoto, Hanmin Zhang and Roger Uhrberg
}

\section{Linköping University Post Print}

\section{Tweet}

N.B.: When citing this work, cite the original article.

Original Publication:

Kazuyuki Sakamoto, Hanmin Zhang and Roger Uhrberg, Adsorption and reaction processes of physisorbed molecular oxygen on Si(111)-(7×7), 2005, Physical Review B. Condensed Matter and Materials Physics, (72), 7, 075346.

http://dx.doi.org/10.1103/PhysRevB.72.075346

Copyright: American Physical Society http://www.aps.org/

Postprint available at: Linköping University Electronic Press

http://urn.kb.se/resolve?urn=urn:nbn:se:liu:diva-50437 


\title{
Adsorption and reaction processes of physisorbed molecular oxygen on $\mathrm{Si}(111)-(7 \times 7)$
}

\author{
Kazuyuki Sakamoto, ${ }^{1, *}$ H. M. Zhang, ${ }^{2}$ and R. I. G. Uhrberg ${ }^{2}$ \\ ${ }^{1}$ Department of Physics, Graduate School of Science, Tohoku University, Sendai 980-8578, Japan \\ ${ }^{2}$ Department of Physics and Measurement Technology, Linköping University, S-581 83 Linköping, Sweden
}

(Received 16 November 2004; published 18 August 2005)

\begin{abstract}
The adsorption and reaction processes of physisorbed oxygen molecules on a $\mathrm{Si}(111)-(7 \times 7)$ surface have been investigated using time-resolved $\mathrm{O} 1 \mathrm{~s}$ core-level photoemission measurements at $45 \mathrm{~K}$. Physisorbed oxygen molecules are only observed at $45 \mathrm{~K}$ and lower temperatures on a $\mathrm{Si}(111)-(7 \times 7)$ surface. At the dosage when the dangling bonds are saturated by chemisorbed oxygen, the coverage of the physisorbed species increases drastically. This result indicates that oxygen species, which are chemisorbed on top of adatoms, modifies the potential energy curve for an oxygen molecule approaching the surface such that physisorbed oxygen molecules are stabilized. Further, the longer lifetime at a higher dosage indicates that an intermolecular force plays a role for the stabilization of this species. Taking these results into account, an oxidation stagedependent gas-surface interaction for an oxygen molecule approaching the $\mathrm{Si}(111)$ surface is suggested.
\end{abstract}

DOI: $10.1103 /$ PhysRevB.72.075346

PACS number(s): 68.43.-h, 68.47.Fg, 82.20.Kh, 79.60.-i

\section{INTRODUCTION}

Oxygen adsorption is a typical system to study in order to learn about the fundamental properties of the reactions of diatomic molecules on surfaces. Together with this scientific interest, the question of how oxygen molecules react on surfaces should be important for applications such as metal oxides in heterogeneous catalysis and semiconductor oxides in device technology. The fundamental steps of oxygen adsorption are considered to be trapping into a physisorbed state that leads to a molecularly chemisorbed state, in which the oxygen molecules are negatively charged and thus have weakened and extended $\mathrm{O}-\mathrm{O}$ bonds. The last step leads to chemisorption in a stable dissociated atomic form. ${ }^{1}$ These adsorption steps are illustrated in Fig. 1(a) by the onedimensional gas-surface interaction potential that a diatomic oxygen molecule approaching a surface feels. $E_{\mathrm{c}}$ and $E_{\mathrm{d}}$ are the potential energy barriers for chemisorption and for dissociation, respectively. [The potential energies of the barriers that are below zero in Fig. 1(a), indicate that the physisorbed and chemisorbed species are metastable. $\left.{ }^{2,3}\right]$ On metal sur-

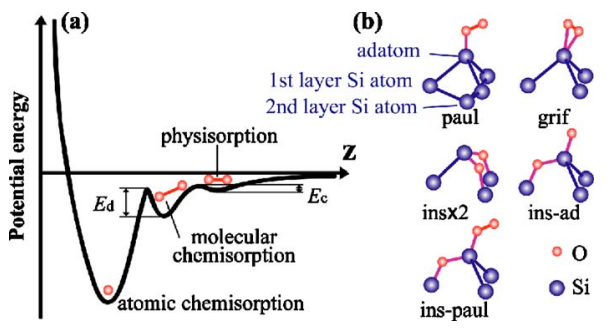

FIG. 1. (Color online) (a) One-dimensional potential energy diagrams illustrating the gas-surface interaction that a diatomic oxygen molecule approaching a surface feels. $Z$ corresponds to the distance from the surface. (b) Schematic illustrations of the bonding configurations of chemisorbed oxygen species discussed in the text. The notations "paul" and "grif" indicate $\mathrm{O}_{2}$ molecules adsorbed on top of adatoms of a $\mathrm{Si}(111)-(7 \times 7)$ surface, and "ad" and "ins" correspond to an $\mathrm{O}$ atom bonding on top of a $\mathrm{Si}$ adatom and to an $\mathrm{O}$ atom inserted into its back bond. faces, the three types of adsorption have been reported both experimentally and theoretically, ${ }^{4,5}$ and in many cases the reaction of oxygen is observed to go through these steps.

Regarding oxygen adsorption on semiconductor surfaces, a great deal of endeavor has been devoted to silicon surfaces. ${ }^{6}$ In contrast to metal surfaces, chemisorbed oxygen molecules were reported to be unstable on a clean $\mathrm{Si}(111)$ $(7 \times 7)$ surface,$^{7-17}$ and to be stabilized only by the presence of atomic oxygen in the form of ins-paul. ${ }^{12-17}$ That is, there is no local minimum in the potential energy diagram for the molecular paul and grif configurations $\left(E_{\mathrm{d}}=0\right)$, and oxygen molecules approaching the $\mathrm{Si}(111)-(7 \times 7)$ clean surface go directly to the atomic ins $\times 2$ and/or ins-ad configurations, ${ }^{7}$ and as a consequence of the presence of these atomic species $E_{\mathrm{d}}$ becomes $\neq 0$. The notations "paul" and "grif" correspond to oxygen molecules adsorbed on top of adatoms of the dimer-adatom-stacking fault structure ${ }^{18}$ of the $\mathrm{Si}(111)$ $(7 \times 7)$ surface, and "ad" and "ins" correspond to an oxygen atom bonding on top of an adatom and an oxygen atom inserted into a back bond, respectively [Fig. 1(b)]. There are many studies on the chemisorbed states, but none report on the adsorption and reaction processes of the physisorbed oxygen molecules on a $\mathrm{Si}(111)-(7 \times 7)$ surface. It has, however, been reported that a physisorbed species shows an interesting reaction process on, for example, a semiconducting $\mathrm{TiO}_{2}$ surface. ${ }^{19}$ Further, although the gas-surface interaction potential is suggested to be different at different oxygen coverage, there is no systematic study of this issue.

In this paper, we present the adsorption and reaction processes of physisorbed oxygen molecules on a $\mathrm{Si}(111)$ $(7 \times 7)$ surface studied by time-resolved high-resolution $\mathrm{O} 1 \mathrm{~s}$ core-level measurements. The coverage of the physisorbed oxygen increases drastically at the dosage when the dangling bonds of the adatoms are saturated with chemisorbed oxygen atoms and/or molecules. This result indicates that the chemisorption of oxygen species on top of the adatoms modifies the potential energy curve for an oxygen molecule approaching the surface by changing $E_{\mathrm{c}}$ to a larger value. Studies of the lifetime of the physisorbed species show that it is more 


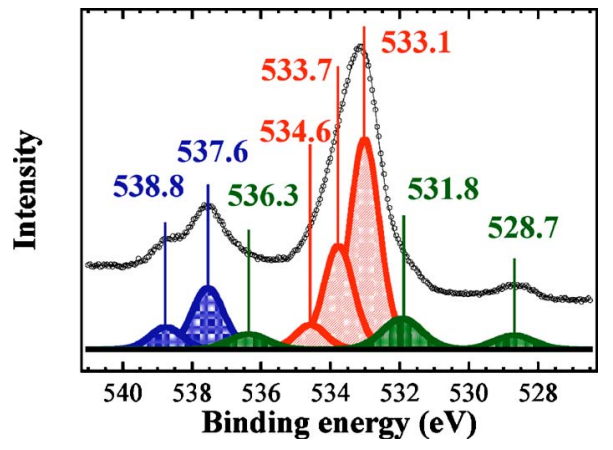

FIG. 2. (Color online) $\mathrm{O} 1 \mathrm{~s}$ core-level spectrum of a $10 \mathrm{~L} \mathrm{O}_{2}$ exposed $\mathrm{Si}(111)-(7 \times 7)$ surface at $45 \mathrm{~K}$, recorded 45 min after the exposure. The open circles are the experimental data, and the solid line overlapping the circles is the fitting result that is obtained using the components shown below the spectrum.

stable just after stopping the exposure, and at higher dosages as well. Since the distance between two oxygen molecules is shorter at a higher coverage and the attractive van der Waals force is stronger at a shorter intermolecular distance, we conclude that the high stability of the metastable physisorbed oxygen molecules originates from the increased attractive intermolecular force. Moreover, the time-dependent intensities of the chemisorbed oxygen species suggest that the dissociation of the physisorbed oxygen molecules produces the oxygen atoms chemisorbed into the back bonds of adatoms and between the first- and second-layer $\mathrm{Si}$ atoms.

\section{EXPERIMENTAL DETAILS}

The core-level photoemission measurements were performed at beamline I311 at the MAX-II synchrotron radiation facility in Lund, Sweden. O $1 s$ core-level spectra were obtained with an angle-integrated photoelectron spectrometer with a total energy resolution of $\sim 0.23 \mathrm{eV}$ at a photon energy of $665 \mathrm{eV}$, and an acceptance angle of $15^{\circ}$. The base pressure was below $4 \times 10^{-11}$ Torr. The $\mathrm{Si}(111)$ sample, cut from an Sb-doped ( $n$-type) Si wafer, was annealed at $1520 \mathrm{~K}$ for $3 \mathrm{~s}$ in the vacuum chamber to obtain a clean surface. After the annealing, a sharp $7 \times 7$ low-energy electrondiffraction pattern was observed, and neither the $\mathrm{C} 1 \mathrm{~s}$ nor the $\mathrm{O} 1 \mathrm{~s}$ peak was detected in the core-level spectra. The $\mathrm{Si}(111)-(7 \times 7)$ sample was then cooled down to $45 \mathrm{~K}$ before the oxygen exposure, i.e., $10 \mathrm{~K}$ above the desorption temperature of condensed $\mathrm{O}_{2}$ multilayers. ${ }^{15,20,21}$ The quality of the $\mathrm{O}_{2}$ gas was confirmed by a quadrupole mass spectrometer.

\section{RESULTS AND DISCUSSION}

Figure 2 shows the $\mathrm{O} 1 \mathrm{~s}$ core-level spectrum of a 10 Langmuir (L; $1 \mathrm{~L}=1 \times 10^{-6}$ Torr $\left.\times \mathrm{s}\right) \mathrm{O}_{2}$ exposed $\mathrm{Si}(111)$ $(7 \times 7)$ surface, recorded $45 \mathrm{~min}$ after the exposure using $h \nu=665 \mathrm{eV}$. Four structures are clearly observed at 528.7, $533.1,537.6$, and $538.8 \mathrm{eV}$. Of these four structures, the $533.1-\mathrm{eV}$ peak is broader than the other peaks, and the 537.6-eV peak has a tail on its lower-binding-energy side. To identify the $\mathrm{O} 1 s$ components that contribute to the spectral shape, we have analyzed the spectrum by a standard leastsquares-fitting method using Voigt line shapes. The solid line overlapping the data points (open circles) is the fitting result. A $200 \mathrm{meV}$ full width at half maximum (FWHM) for the Lorentzian contribution and Gaussian widths of 850-920 meV FWHM's were used for all $\mathrm{O} 1 s$ components that are shown below the spectrum. From the results of the fitting procedure, we realize that the broad 533.1-eV peak originates from four $\mathrm{O} 1 \mathrm{~s}$ components, whose binding energies are $531.8,533.1,533.7$, and $534.6 \mathrm{eV}$, and that the tail of the $537.6-\mathrm{eV}$ peak results from the presence of a component at $536.3 \mathrm{eV}$. Based on the relative binding energies of the $\mathrm{O} 1 \mathrm{~s}$ core-level components reported in the former studies, ${ }^{16,17,22,23}$ we conclude that the three at 533.1, 533.7, and $534.6 \mathrm{eV}$ (the relative binding energies are 0.0, 0.6, and $1.5 \mathrm{eV}$ ) originate from oxygen atoms chemisorbed into the back bonds of adatoms, between the first- and second-layer $\mathrm{Si}$ atoms, and on top of the adatoms, respectively, and that the three at 528.7, 531.8, and $536.3 \mathrm{eV}$ (the relative binding energies are $-4.4,-1.3$, and $3.2 \mathrm{eV}$ ) result from the ins-paul species. In contrast to these six components, the 537.6- and $538.8-\mathrm{eV}$ structures were not observed in the former studies performed between 100 and $300 \mathrm{~K}$, nor in the spectra obtained after annealing the sample at $600 \mathrm{~K}$. Taking into account that chemisorbed oxygen species with all possible bonding configurations were observed in these former studies, the 537.6-eV and 538.8-eV structures cannot originate from chemisorbed oxygen species.

The oxygen molecule has an open shell in its ${ }^{3} \Sigma$ ground state, and photoionization from the $\mathrm{O} 1 \mathrm{~s}$ core-level leads to two multiplet components $\left({ }^{4} \Sigma\right.$ and $\left.{ }^{2} \Sigma\right)$ in the gas phase, i.e., the paramagnetic splitting that originates from two final states with different spins. ${ }^{24}$ In the case of oxygen molecules physisorbed on a graphite surface, this paramagnetic splitting has been reported to be preserved and to have a value of 1.2 eV. ${ }^{25}$ Further, the ${ }^{4} \Sigma$ component (the stronger component of the doublet) was observed at binding energies between 537.4 and $537.7 \mathrm{eV}$ depending on the oxygen coverage on a graphite surface. Taking the similarities in both the binding energies and splitting into account, we conclude that the 537.6and 538.8-eV structures originate from the ${ }^{4} \Sigma$ and ${ }^{2} \Sigma$ components of physisorbed oxygen molecules.

In order to investigate the adsorption process of the physisorbed oxygen molecules on a $\mathrm{Si}(111)-(7 \times 7)$ surface, we consider the dosage-dependent intensities of the $\mathrm{O} 1 \mathrm{~s}$ components. Figure 3(a) shows the normalized dosage-dependent intensity of the 537.6-eV component together with those of the 533.1-, 534.6-, and 528.7-eV components, and (b) displays the dosage-dependent intensities of the 528.7-, 533.1-, 534.6-, and 537.6-eV O 1 s component up to $4 \mathrm{~L}$. The intensity of each component is normalized with its maximum intensity in (a). The intensities in Fig. 3 are obtained by analyzing the photoemission spectra of the $\mathrm{Si}(111)$ surface exposed to ambient $\mathrm{O}_{2}$ pressures of $1 \times 10^{-9}$ Torr at dosages up to $3 \mathrm{~L}$ and of $5 \times 10^{-9}$ Torr at dosages above $3 \mathrm{~L}$. The acquisition time of one photoemission spectrum was approximately $30 \mathrm{~s}$, i.e., the difference in time between one data point and the next is approximately $30 \mathrm{~s}$ in Fig. 3. Here we note that the intensity of the 538.8-eV component showed 


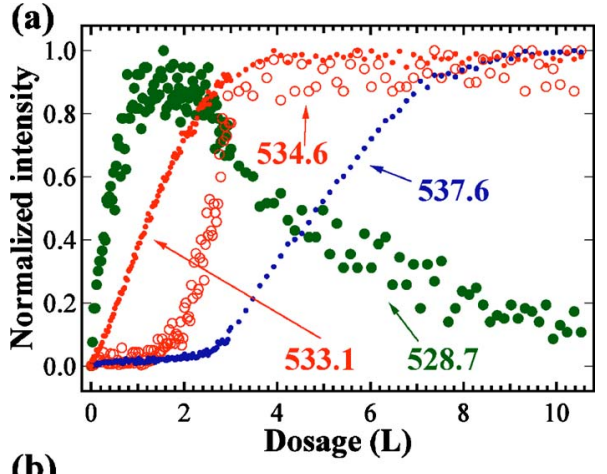

(b)

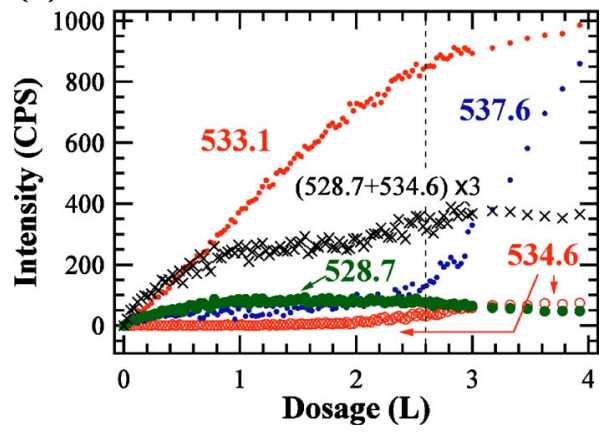

FIG. 3. (Color online) (a) Normalized dosage-dependent intensities of the 528.7-, 533.1-, 534.6-, and 537.6-eV O $1 s$ components. The intensity of each component is normalized with its maximum intensity in (a). (b) Dosage-dependent intensities of the 528.7-, 533.1-, 534.6-, and 537.6-eV O 1 s component up to $4 \mathrm{~L}$. The crosses in (b) are the sum of the intensities of the 528.7- and 534.6-eV components, which are multiplied by 3 . The intensities in (a) and (b) are obtained by continuously measuring photoemission spectra of the $\mathrm{Si}(111)$ surface exposed to ambient $\mathrm{O}_{2}$ pressures of $1 \times 10^{-9}$ Torr at dosages up to $3 \mathrm{~L}$ and of $5 \times 10^{-9}$ Torr at dosages above $3 \mathrm{~L}$. The difference in time between one data point and the next is approximately $30 \mathrm{~s}$.

the same dosage dependence as that of the 537.6-eV component, the dosage dependence of the 533.7-eV component was similar to that of the 534.6-eV component, and the dosagedependent intensities of the three components that originate from the ins-paul species were the same. We also notice that the slope of the intensity increase of the 537.6-eV component is steeper than the slope of the intensity decrease of the 528.7-eV component, i.e., they do not display the exact opposite behavior.

As shown in Fig 3, the rate of intensity increase of the 537.6-eV component changes dramatically at $2.6 \mathrm{~L}$, i.e., the dosage indicated by a dashed line. This dosage corresponds to the dosage where the sum of the intensities of the 528.7and 534.6-eV components [the crosses in Fig. 3(b)] almost saturates. Since the 528.7-eV component originates from the ins-paul species, and the 534.6-eV component results from oxygen atoms adsorbed on top of the adatoms, all dangling bonds of the $\mathrm{Si}(111)-(7 \times 7)$ surface should be saturated at 2.6 L. Therefore, we conclude that the physisorbed oxygen molecule is rather unstable on a clean $\mathrm{Si}(111)-(7 \times 7)$ surface, but its stability becomes higher on a surface on which oxygen atoms and/or molecules are already adsorbed at the on-top sites of the adatoms. This means that an oxygen spe-

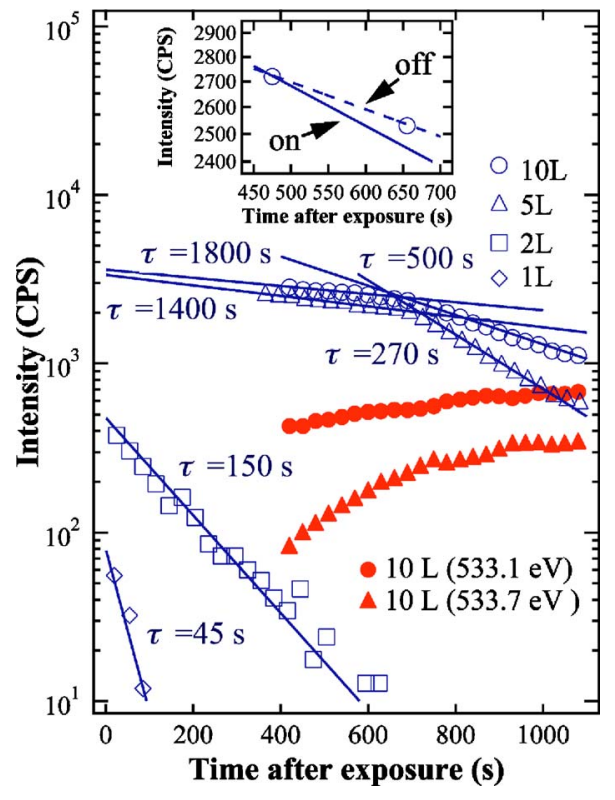

FIG. 4. (Color online) Time dependences of the O $1 s$ core-level intensities for the physisorbed oxygen molecules (the sum of the intensities of the 537.6- and 538.8-eV components) of 1, 2, 5, and $10 \mathrm{~L}$ oxygen exposed surfaces (open symbols), and the 533.1- and 533.7-eV components of a $10 \mathrm{~L}$ oxygen exposed surface (filled symbols). The inset shows the time-dependent intensities of the $\mathrm{O}$ $1 s$ core-level of the physisorbed oxygen of a $10 \mathrm{~L}$ exposed surface obtained under continuous photon irradiation (solid line), and by turning off the photon irradiation for a period (photon irradiation was turned off between measuring the open circles).

cies, which is chemisorbed on top of an adatom, modifies the potential energy curve that an oxygen molecule approaching the surface feels, such that $E_{\mathrm{c}}$ changes to a larger value. This kind of change was also observed for physisorbed nitrogen molecules on a $\mathrm{Ru}(001)$ surface. $^{26}$ That is, on a $\mathrm{Ru}(001)$ surface, the physisorbed nitrogen molecules are reported to be unstable on a clean surface, and to be stabilized only by the presence of a certain amount of chemisorbed nitrogen molecules.

The reaction process of the physisorbed molecular oxygen has been investigated by measuring the time dependence of the $\mathrm{O} 1 \mathrm{~s}$ core-level intensities. Figure 4 shows the time dependence of the integrated intensities of the $\mathrm{O} 1 \mathrm{~s}$ components for the physisorbed molecular oxygen (the sum of the intensities of the 537.6- and 538.8-eV components). Data for $1,2,5$, and $10 \mathrm{~L}$ oxygen exposed $\mathrm{Si}(111)$ surfaces are shown by open symbols. The time dependences of the 533.1- and 533.7-eV components of a $10 \mathrm{~L}$ exposed surface are shown by filled symbols. The results in Fig. 4 were obtained by normalizing the spectra using the background intensities that are proportional to the photon flux. As can be seen from the figure, the $\mathrm{O} 1 s$ intensity from the physisorbed oxygen molecule decreases with time, and the $\mathrm{O} 1 \mathrm{~s}$ intensities of both the 533.1- and 533.7-eV components increase on a $10 \mathrm{~L}$ exposed surface. The time-dependent intensity increases of the 533.1- and 533.7-eV components were also observed on a $5 \mathrm{~L}$ exposed surface. In contrast to this behavior, the intensities of the $\mathrm{O} 1 s$ components of the ins-paul species and the 
534.6-eV component hardly changed with time on 1, 2, 5, and $10 \mathrm{~L}$ oxygen exposed surfaces. These results indicate that the reaction path of physisorbed oxygen molecules produced atomic oxygen chemisorbed into the back bonds of adatoms and between the first- and second-layer Si atoms. Further, the negligible time-dependent change in intensity of the ins-paul oxygen species, which has been reported to have finite lifetimes at temperatures between 100 and $300 \mathrm{~K},{ }^{14-17}$ indicates that this species is quite stable at $45 \mathrm{~K}$. Thus the origin of the intensity decrease of the 528.7-eV component observed in Fig. 3 is not a time effect. In Fig. 3, the intensity of the 528.7-eV component starts to decrease at $2 \mathrm{~L}$, i.e., a dosage where the intensity of the 533.1-eV component is not saturated and the intensities of the 533.7-, 534.6-, and 537.6-eV components are still small. The small intensities indicate that the physisorbed species, and the oxygen atoms chemisorbed on top of adatoms and between the first- and second-layer Si atoms, hardly contribute to the decrease of the ins-paul species. Taking into account that a different number of ins oxygen atoms per adatom should modify the charge state of the adatom and thus the amount of charge transferred into the $2 \pi^{*}$ antibonding molecular orbital, we propose that a larger number of chemisorbed oxygen atoms promotes the dissociation of the ins-paul species by changing $E_{\mathrm{d}}$ to a value close to $0\left(E_{\mathrm{d}} \sim 0\right)$ on the time scale of the present study (30 s). Here we notice that although the intensities of the $\mathrm{O} 1 s$ peaks can be affected by photoelectron diffraction effect, such effect has been found to be negligible for the $\mathrm{O} 1 \mathrm{~s}$ components of the physisorbed molecule and those of the 533.1- and 533.7-eV components.

By fitting the intensity decreases using the equation $y$ $=y_{0} \exp (-x / \tau)$, where $y_{0}$ is the intensity of the $\mathrm{O} 1 \mathrm{~s}$ corelevel of the physisorbed oxygen just after stopping the exposure, we can derive the lifetime $(\tau)$ of the physisorbed oxygen molecules. The solid lines that overlap the $\mathrm{O} 1 \mathrm{~s}$ intensities of the physisorbed oxygen molecules on the 1 and $2 \mathrm{~L}$ exposed $\mathrm{Si}(111)$ surfaces in Fig. 4 result from $\tau=45 \mathrm{~s}$ and $150 \mathrm{~s}$, respectively. On the 5 and $10 \mathrm{~L}$ oxygen exposed $\mathrm{Si}(111)$ surfaces, the $\mathrm{O} 1 s$ core-level intensities of the physisorbed oxygen molecules do not show simple exponential decays. First the intensities decrease slowly (first region), and then the decrease rates become larger (second region). Regarding the $5 \mathrm{~L}$ exposed surface, the lifetimes are found to be $1400 \mathrm{~s}$ and $270 \mathrm{~s}$ in these two regions, and those on the 10 $\mathrm{L}$ exposed surface to be $1800 \mathrm{~s}$ and $500 \mathrm{~s}$.

All these lifetimes were obtained under photon irradiation, and photon irradiation generally dissociates and/or desorbs weakly adsorbed physisorbed species. In order to discuss the influence of photon irradiation, we have measured the intensity decreases of the $\mathrm{O} 1 s$ components for the physisorbed molecular oxygen by turning off the photon irradiation for a period. The inset of Fig. 4 shows the intensity decrease obtained for a $10 \mathrm{~L}$ exposed surface by turning off the irradiation for a certain time (photon irradiation was turned off between measuring the open circles shown in the inset). As shown in the figure, the intensity decrease of the open circles is slower than that of the solid line, which represents the result obtained under continuous photon irradiation. By fitting the intensity decrease of the open circles (dashed line), the lifetime is found to be 2800 s, i.e., a value that is approximately 1.6 times longer than the lifetime obtained by irradiating the surface continuously. In the second region of the $10 \mathrm{~L}$ oxygen exposed surface, the lifetime obtained by eliminating the irradiation effect was $550 \mathrm{~s}$. Regarding the $5 \mathrm{~L}$ exposed surface, the influences of photon irradiation have been found to be approximately the same as those on the $10 \mathrm{~L}$ exposed surface in each region. These results indicate that approximately $1 / 3$ of the reaction of the physisorbed oxygen species results from the influence of photon irradiation in the first region of the 5 and $10 \mathrm{~L} \mathrm{ex-}$ posed surfaces, and that thermal activation is the dominant effect of reaction in the second region.

In order to discuss the reaction path of the physisorbed oxygen molecules in more detail, we compare the $\mathrm{O} 1 \mathrm{~s}$ intensity decrease with the intensity increases of the 533.1- and 533.7-eV components. On the $10 \mathrm{~L}$ exposed $\mathrm{Si}(111)$ surface, the intensity of the physisorbed oxygen decreases approximately 190 counts per second (CPS), and the intensities of the 533.1- and 533.7-eV components increase approximately $45 \mathrm{CPS}$ and 60 CPS in the first region in the case of eliminating the irradiation effect. The intensity changes correspond to the change in coverage of each species, and by assuming that a physisorbed oxygen molecule only produces two chemisorbed oxygen atoms by dissociation or leads to a desorption from the surface, one can estimate that $\sim 45 \%$ of the physisorbed oxygen molecules desorb, and of the $\sim 55 \%$ of the physisorbed molecules that dissociate, $\sim 42 \%$ transform into oxygen atoms adsorbed into the back bonds of adatoms and $\sim 58 \%$ adsorb between the first- and secondlayer $\mathrm{Si}$ atoms. On the $5 \mathrm{~L}$ exposed surface, the intensity decreases of the physisorbed oxygen and the intensity increases of the chemisorbed atomic oxygen indicated that $\sim 24 \%$ dissociates in the first region. Here we notice that this time-dependent reaction of the physisorbed oxygen molecules is not inconsistent with the result shown in Fig. 3. The physisorbed oxygen molecules, which cover the surface, might obstruct the chemisorption of the next incoming oxygen molecule, and the $\mathrm{O} 1 \mathrm{~s}$ core-level intensities of the chemisorbed species would be saturated even if vacant sites for chemisorption remain. Further, since the result shown in Fig. 3 is obtained under oxygen dosage while the result in Fig. 4 is obtained after stopping the exposure, the physisorbed oxygen molecules might always be condensed in Fig. 3 but not in the result shown in Fig. 4. Thus, by assuming that physisorbed oxygen molecules hardly dissociate when they are condensed but they dissociate easier when the neighboring molecule is desorbed, the time-dependent reaction products of the physisorbed oxygen can be adsorbed on the vacant sites mentioned above.

The lifetimes shown in Fig. 4 indicate that the physisorbed oxygen molecules are more stable until approximately $700 \mathrm{~s}$ after stopping the exposure, and at higher dosages. The lower stability of the physisorbed oxygen molecules on the 1 and 2 L exposed $\operatorname{Si}(111)$ surfaces, and the higher stability on the 5 and $10 \mathrm{~L}$ exposed surfaces observed in the first region, fit well with the results shown in Fig. 3, i.e., small intensities below $2.6 \mathrm{~L}$ and large intensities above $2.6 \mathrm{~L}^{27}$ This means that the difference in the lifetime of the physisorbed oxygen molecules obtained just after stopping the exposure results from the modification of the potential 
energy curve by oxygen species chemisorbed on top of adatoms. In contrast to this case, the change in stability in the two regions observed on the 5 and $10 \mathrm{~L}$ exposed $\mathrm{Si}(111)$ surfaces cannot be explained by the same origin, since both the intensity of the ins-paul species and the intensity of the $534.6-\mathrm{eV}$ component hardly changed with time. The differences between these two surfaces are the coverage of physisorbed oxygen molecules and the coverage of chemisorbed species produced by the dissociation of the physisorbed species. According to the reaction discussed above, the coverage of chemisorbed species produced by the dissociation of the physisorbed species is smaller on a $5 \mathrm{~L}$ exposed $\mathrm{Si}(111)$ surface than that on a $10 \mathrm{~L}$ exposed surface. This suggests that the effect of reaction products should be smaller on the $5 \mathrm{~L}$ exposed surface, though the lifetime is shorter and the difference in lifetime between the first and second region is larger on the $5 \mathrm{~L}$ exposed surface. Thus the chemisorbed species produced by the dissociation of the physisorbed species cannot be the origin of the change in stability observed in Fig. 4. The distance between two oxygen molecules is shorter at higher coverages and the attractive van der Waals force becomes stronger until the intermolecular distance becomes as short as the distance in solid oxygen, i.e., as long as the total energy gain compensates the increasing mutual repulsion. This explains well the results shown in Fig. 4, and we therefore conclude that the higher stability of the metastable physisorbed oxygen molecules observed at a higher coverage originates from a stronger attractive intermolecular force. ${ }^{28}$ This conclusion suggests that there are two origins for the stabilization of physisorbed oxygen molecules, i.e., the saturation of dangling bonds and the intermolecular interactions.

Moreover, since a higher dissociation probability is obtained at a higher physisorbed oxygen coverage, where the physisorbed species is more stable, we propose that the attractive intermolecular force prevents the desorption by changing the potential energy barrier $E_{\mathrm{c}}$ as shown in Figs. 5(c) and 5(d). This proposition is supported by the higher desorption probabilities obtained in the second region ( $\sim 77 \%$ and $\sim 87 \%$ on the 10 and $5 \mathrm{~L}$ exposed surfaces, respectively), since shorter lifetimes suggest that the attractive intermolecular forces are weaker than those in the first region.

\section{CONCLUSION}

In conclusion, we have investigated the adsorption and reaction processes of physisorbed oxygen molecules on a

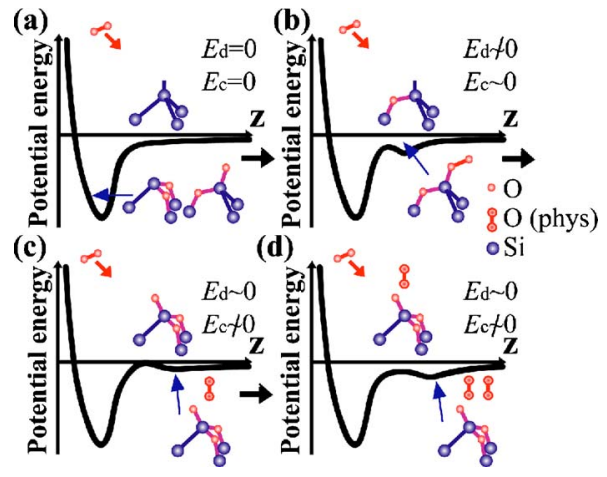

FIG. 5. (Color online) One-dimensional potential energy diagrams illustrating the gas-surface interaction that an oxygen molecule approaching a $\mathrm{Si}(111)-(7 \times 7)$ surface feels on the time scale of the present study. (a)-(d) show the potential energy diagrams at different stages of oxidation.

$\operatorname{Si}(111)-(7 \times 7)$ surface using time-resolved high-resolution O $1 s$ core-level photoelectron measurements. The coverage of the physisorbed oxygen molecules increases drastically at the dosage when all adatoms have oxygen species at their on-top sites. This result indicates that $E_{\mathrm{c}}$ is almost 0 on the time scale of the present study and physisorbed oxygen molecules are hardly stable on a surface with dangling bonds [Figs. 5(a) and 5(b)], and that $E_{\mathrm{c}}$ changes to a larger value when the dangling bonds become saturated by chemisorbed oxygen, as indicated in Fig. 5(c). As the number of oxygen atoms adsorbed in the back bonds of adatoms increases, $E_{\mathrm{d}}$ becomes almost 0 on the time scale of the present study, and the dissociation of the ins-paul species is promoted [Fig. 5(c)]. The different lifetimes obtained at different dosages suggest that the attractive van der Waals force between two molecules changes the potential energy barrier $E_{\mathrm{c}}$ at a high coverage of physisorbed molecular oxygen. This promotes the dissociation into atomic oxygen that chemisorbs into the back bonds of adatoms and between the first- and secondlayer $\mathrm{Si}$ atoms [Fig. 5(d)].

\section{ACKNOWLEDGMENTS}

Experimental support from Dr. J. Weissenrieder and the MAX-lab staff and fruitful discussion with Dr. F. Matsui are gratefully acknowledged. This work was financially supported by the Swedish Research Council. K.S. was partially supported by the MEXT 21st century COE Program "Exploring New Science by Bridging Particle-Matter Hierarchy."

\footnotetext{
*Electronic address: sakamoto@surface.phys.tohoku.ac.jp

${ }^{1}$ G. Somorjai, Introduction to Surface Chemistry and Catalysis (Wiley, New York, 1994).

${ }^{2}$ A. Zangwill, Physics at Surfaces (Cambridge, New York, 1988).

${ }^{3}$ A molecule approaching the surface loses energy by, e.g., exciting surface phonons, and thus can stay in a state with a barrier whose potential energy is below zero.
}

${ }^{4}$ See, for example, G. R. Darling and S. Holloway, Rep. Prog. Phys. 58, 1595 (1995).

${ }^{5}$ E. J. Walter, S. P. Lewis, and A. M. Rappe, J. Chem. Phys. 113, 4388 (2000), and references therein.

${ }^{6}$ See, for example, T. Engel, Surf. Sci. Rep. 18, 91 (1993).

${ }^{7}$ S.-H. Lee and M.-H. Kang, Phys. Rev. Lett. 82, 968 (1999); Phys. Rev. B 61, 8250 (2000); Phys. Rev. Lett. 84, 1724 (2000). 
${ }^{8}$ T. Hoshino and Y. Nishioka, Phys. Rev. B 61, 4705 (2000).

${ }^{9}$ K.-Y. Kim, T.-H. Shin, S.-J. Han, and H. Kang, Phys. Rev. Lett. 82, 1329 (1999).

${ }^{10}$ G. Comtet, L. Hellner, G. Dujardin, and K. Bobrov, Phys. Rev. B 65, 035315 (2002).

${ }^{11}$ A. J. Mayne, F. Rose, G. Comtet, L. Hellner, and G. Dujardin, Surf. Sci. 528, 132 (2003).

${ }^{12}$ K. Sakamoto, S. Doi, Y. Ushimi, K. Ohno, H. W. Yeom, T. Ohta, S. Suto, and W. Uchida, Phys. Rev. B 60, R8465 (1999).

${ }^{13}$ K. Sakamoto, S. T. Jemander, G. V. Hansson, and R. I. G. Uhrberg, Phys. Rev. B 65, 155305 (2002).

${ }^{14}$ T. Jensen, L.-B. Tækker, C. Gundlach, F. K. -Dam, P. Morgen, S. V. Hoffman, Z. Li, and K. Pedersen, Phys. Rev. B 64, 045304 (2001).

${ }^{15}$ F. Matsui, H. W. Yeom, K. Amemiya, K. Tono, and T. Ohta, Phys. Rev. Lett. 85, 630 (2000).

${ }^{16}$ K. Sakamoto, F. Matsui, M. Hirano, H. W. Yeom, H. M. Zhang, and R. I. G. Uhrberg, Phys. Rev. B 65, 201309(R) (2002).

${ }^{17}$ K. Sakamoto, H. M. Zhang, and R. I. G. Uhrberg, Phys. Rev. B 68, 075302 (2003).

${ }^{18}$ K. Takayanagi, Y. Tanishiro, M. Takahashi, and S. Takahashi, Surf. Sci. 164, 367 (1985)

${ }^{19}$ E. Wahlström, E. K. Vestergaard, R. Schaub, A. Ronnau, M. Vestergaard, E. Lægsgaard, I. Stensgaard, and F. Besenbacher, Science 303, 511 (2004).

${ }^{20}$ A. J. Schell-Sorokin and J. E. Demuth, Surf. Sci. 157, 273
(1985).

${ }^{21}$ C. Silvestre, J. Hladky, and M. Shayegan, J. Vac. Sci. Technol. A 8, 2743 (1990).

${ }^{22}$ U. Höfer, P. Morgen, W. Wurth, and E. Umbach, Phys. Rev. Lett. 55, 2979 (1985); Phys. Rev. B 40, 1130 (1989).

${ }^{23}$ B. Lamontagne, D. Roy, R. Sporken, and R. Caudano, Prog. Surf. Sci. 50, 315 (1995).

${ }^{24}$ M. Larsson, P. Baltzer, S. Svensson, B. Wannberg, N. Mårtensson, A. Naves de Brito, N. Correia, M. P. Keane, M. CarlssonGöthe, and L. Karlsson, J. Phys. B 23, 1175 (1990).

${ }^{25}$ A. Nilsson, R. E. Palmer, H. Tillborg, B. Hernnäs, R. J. Guest, and N. Mårtensson, Phys. Rev. Lett. 68, 982 (1992).

${ }^{26}$ P. Feulner and D. Menzel, Phys. Rev. B 25, R4295 (1982).

${ }^{27}$ The original lifetimes of 1 and $2 \mathrm{~L}$ exposed surfaces were not measured by eliminating the influence of photon irradiation. However, since the influence of photon irradiation was almost constant on the 5 and $10 \mathrm{~L}$ exposed surfaces, thermal activation should be the dominant effect on 1 and 2 L exposed surfaces, and the original lifetimes should be close to the values indicated in Fig. 4.

${ }^{28}$ At low coverages, most of the metastable physisorbed oxygen molecules would desorb and/or dissociate before feeling the attractive interaction of another oxygen species due to its finite lifetime. Thus this conclusion does not suggest the formation of islands at the very beginning of adsorption. 